fit during the temporary delay, but with reasonable assurance that the patient will, as the result of its use, be the better able to undergo the graver operation. Again, it may be done by an assistant at the same time that delivery is being made, and by thus ridding the system of toxins hasten the convalescence and obviate the danger of a continuance of the convulsions after delivery.

Veratrum viride, without doubt the most reliable of all remedies in the so-called expectant treatment, is supposed to act by powerfully depressing the circulation, and thereby bleeding the woman into her own vessels; it is also supposed to reduce the spasm of the renal vessels, causing an increased flow of urine and encouraging perspiration by relieving spasm of the capillaries.

If this theory is correct, the effects are; to an extent, exactly what we hope to obtain by the venesectioninfusion method, with the difference that by the latter, instead of the patient being bled into her own vessels and retaining the cause of the trouble within the system, the poisoned blood is withdrawn and replaced with salt solution, thereby promoting diuresis, diaphoresis, etc., and what is most important, in many of these cases, stimulation of the heart and vasomotor centers, which is directly the opposite to the ultimate effect of veratrum viride. It is also worthy of note that while veratrum viride is supposed to control the convulsion by depressing the circulation, the venesection-infusion treatment, although temporarily reducing arterial pressure, finally stimulates the circulation and raises blood-pressure; yet, as demonstrated by my cases, the convulsions cease.

Hot baths, for the purpose of encouraging perspiration, used as a preventive before the convulsions appear, and to guard against recurrence after they have ceased, are unquestionably a valuable aid to other means, but for direct relief of the convulsions they are obviously too slow. It may even be seriously questioned whether by rapidly drawing large quantities of liquid from the blood by a process approaching filtration, an. abnormal proportion of the more insoluble products (possibly toxins) are not left behind; thus instead of the toxins being eliminated they become more concentrated, consequently more irritating. 'The indications for, and the effect of, hydragogue cathartics are practically the same as those of hot baths and these cathartics can be advantageously used at the same time.

Considering the cause, as we understand it to-day, morphin is theoretically contra-indicated in all cases of puerperal eclampsia, for the reason that it inhibits both secretion and excretion, thus locking up the toxins in the system, and when used in doses large enough to control the convulsions undoubtedly endangers the life of both mother and child by prolonging the posteclamptic coma. In emergencies, when other means are not at hand, its use is justifiable.

Chloroform, the most generally useful of all means at our disposal for the treatment of puerperal eclampsia, should be used to control the convulsions while waiting for the less active remedies to take effect, and for anesthesia when the more radical means are employed; but as recent investigations have shown that its prolonged use causes acute degeneration of the liver and kidney cells, it is advisable, on account of the already crippled condition of these organs, to substitute ether when it is necessary to continue the administration for a considerable time.

490 Endicott Arcade.

\section{THE SITUATION AS REGARDS SALVARSAN (606)}

\author{
WILLTAM ALLEN PUSEY, M.D. \\ CHICAgo
}

Indications at present are very strong that we are on the verge of a period of indiscriminate and reckless use of Ehrlich's new remedy, 606, or salvarsan, as it is known commercially, that will result in disappointment in that valuable remedy and-what is more importantin damage to many syphilitic patients, chiefly, let us hope, from neglect of established measures of treatment. Indeed, it will be very fortunate if we do not have another therapeutic fiasco, like that following the announcement of Koch's tuberculin, in which a valuable remedy failed to come up to first expectations, and thereby caused disappointment from which it did not recover for ten years. For in the excitement over 606 we are apparently remembering only that it was introduced to cure syphilis, and are therefore assuming that it will cure it, and are not taking heed of the fact that experience already is showing that it is not doing it. Undoubtedly the basis of the sensational interest in 606 - the sole basis in the popular, if not in the professional, mind-is the belief that the new remedy is a cure for syphilis; that it will completely destroy the infection and leave the patient free from the disease; that the person who gets syphilis will be cured "at one shot"-to adopt an expression concerning 606 now in the vernacular; and that the disease will be robbed of its seriousness. Take, for example, the following statement ${ }^{1}$-not from the daily press, but from one of the soberest of scientific journals: "One of the greatest scourges of humanity-perhaps the most insidious and cruel of all, since it so often places its victims beyond the pale of human sympathy, to be loathed rather than pitied-is on the point of being eradicated." The patient is to be cured of syphilis by one injection, or mayhap two or three, and the treatment is so efficient that syphilis is possibly "on the point of being eraủicated."

And this conception of the possibilities of the agent are in accordance with the purpose for which 606 was introduced. 'To cure syphilis was Ehrlich's noble aim. The new drug was to be a therapia sterilisans magnato destroy by one massive dose of a "parasitotropic" remedy all of the infecting organisms in a syphilitic patient.

In the light of even the brief present experience with 606 , it may be said with confidence that the agent has failed in this magnificent aim. It is not a therapia sterilisans magna; it does not destroy the infection; and it does not rid the syphilitic patient of his syphilis. This is not saying that the drug is not a useful remedy; there seems every reason to believe that it is a valuable addition to our means of combating syphilis.

Ehrlich's hopes for 606 were based on animal experiments. Ehrlich recognized, as do others, that experiments on syphilized animals with the agent were not conclusive for man, and he turned over the remedy to clinicians for trial in human syphilis. And it is with the results in human syphilis that we have to do.

The belief that salvarsan cures syphilis in man depends on the following considerations:

5. Schweitzer, H.: Ehrlich's Chemotheraps, Sclence, Dec. 9, 1910, p. 809 . 
1. The destruction of the spirochetes.

2. The reversal of the Wassermann reaction.

3. The removal of the clinical manifestations of syphilis.

The evidence is becoming increasingly strong that the agent does not permanently and completely cause any of these results.

It has a very striking effect on spirochetes, but the sudden disappearance of spirochetes from lesions is no evidence of an overwhelming attack upon the disease. The drug may cause the disappearance of spirochetes from a chancre within twenty-four hours and greatly reduce the number, or cause their disappearance from deeper lesions-but mercury may do the same thing. A little calomel powder dusted on a chancre will within twenty-four hours make it exceedingly difficult or impossible to demonstrate spirochetes; and as soon as a syphilitic patient is brought thoroughly under the influence of mercury the difficulty of demonstrating spirochetes is enormously increased. And yet under such conditions, who attaches any weight to these findings as an evidence of the cure of syphilis? If one were to take any warning from the accumulated experience of generations in syphilis, it would lead him to expect that the apparent disappearance of the spirochetes was but a lull in the invasion and that they would return. And that is exactly what is coming to light. Disappearing spirochetes are returning, it may be, even at the site of the original lesion, where their disappearance has been regarded as of such significant importance.

The Wassermann reaction is very much more reliable evidence of the effect on the disease, for its findings cover the patient's whole organism. Present experience indicates that a reversal to negative occurs in a minor proportion of cases; but if it were made negative in all cases, only time-and much more time than the few weeks after treatment of most of the reports-would show that this negative reaction meant cure. The Wassermann reaction can be made negative by vigorous mercurial treatment-not so readily, however, as by 606 -but no conservative syphilographer regards this as evidence of cure. On the contrary, experience with mercury shows that a return to positive is to be expected, and that treatment must be persisted in after the manner that we have learned from clinical experience. As a matter of fact, we do not yet know how to interpret the reversal of the Wassermann reaction in its bearing on treatment. The same return to positive of cases which have become negative under salvarsan is occurring in the same way as after the use of mercury.

But, after all, the most important evidence as to the value of 606 is the effect on the clinical manifestations of syphilis; and here experience indicates great variability. These variations extend from cases which are "refractory" to the drug, and show no effect, to cases in which strikingly good results are seen. As a rule, there is in active early syphilis - the stage at which most would be expected from a remedy that cured-distinct and positive improvement. Sometimes the symptoms entirely disappear-as happens from mercury or even without treatment-but that, even in these cases, no cure is obtained, is shown by the definite tendency to recurrence that the later literature is revealing.

In fact, accumulating experience simply shows that the remedy has as a rule a distinctly beneficial effect on the lesions of syphilis, but not so positive or beneficial an effect as would give any ground for the hope that it will cure the disease by the radical destruction of the invading spirochetes. How definite and constant these effects will be, or whether the action of the drug will be as reliable as that of mercury, cannot with our present experience be determined. But it can at leastand unfortunately-be said from present experience that the effects on symptoms are such as to show that salvarsan falls short of cure of the disease.

How useful 606 will prove is another question. The burden of reports is that it shows marked effects in intractable and destructive lesions. In the usual cases of active early syphilis it is very much to be doubted if it is of more value than mercury. In late lesionslesions of the tertiary period-particularly in those which have been intractable to other treatment, the most striking results have occurred. In the cases of active secondary syphilis of the ordinary types that I have seen treated with salvarsan the effects have certainly not been more rapid or better than would be expected from mercury-not, I believe, so good. In three or four cases with very extensive ulcerative lesions the effects have been remarkably good. Various syphilographers are calling attention to the fact that men are losing sight of the results obtained from mercury, and that the new agent does not show results to indicate any probability of its superseding or being superior to mercury in the treatment of syphilis; that, so far as we can judge from present experience, mercury must still be our reliance, but that as a reserve we have a valuable agent in 606 .

Of the dangers of salvarsan we are least able to speak at present. We do not fully know them. The evidence is large that immediate risk of serious accidents from the remedy are small. But enough is known to show that dangers exist. There is good ground for the belief that a larger proportion of serious accidents are occurring than would be estimated from the present literature. And that serious accidents should occur is not surprising. Salvarsan is the lineal successor of atoxyl, soamin, and arsacetin. All of these were introduced as safe arsenical preparations-atoxyl, so safe that its atoxic character was "blown" as it were in its name-and all have a train of arsenical fatalities and optic atrophies in the wake of their use in therapeutic doses. Salvarsan has about 35 per cent. arsenic content; it is administered in an average dose of half a gram-8 grains-that is, a dose of 2.8 grains of arsenic. There is no doubt that such a dose of arsenic has in it immediate possibilities of optic atrophies and other dangers. The dangers of its use, however, are as nothing in the face of a severe syphilitic crisis, or if the remedy were able to cure syphilis.

There is great diversity of opinion about technic of efficient administration, with much striving for a new technic that will be more effective. Injections in neutral emulsion, in alkaline solution, or mixed with oil, into the subcutaneous tissue, into the muscles, or into the veins, or combinations of these various methods of administration are succeeding each other. The hope of a therapia sterilisans magna-the complete destruction of the spirochetes of syphilis in an infected patient-is practically abandoned, and two or three or more injections are being used. And, finally, the recommendation of the use of salvarsan and then mercury, as heretofore, is the last evidence that the new agent is not equal to its proposed mission.

It cannot be emphasized too strongly that the situation with 606 is still experimental-and more experimental 
than it was thought to be when the drug was introduced. Its position is not established; the degree of its usefulness-even of its immediate usefulness on the active manifestations of the disease-is not established. The amount of beneficial effect it will have on the afterhistory of syphilis is, of course, with our present brief experience with 606 , purely theoretical-with grounds existing for different theories. Our present experience shows that it does not cure syphilis and that we are not justified in holding out to patients any hope of cure by it, but that it is likely to prove a useful remedy in syphilis, with mercury, however, as before, our chief dependence.

The foregoing statement can be abundantly substantiated from the recent literature. Particular attention in this connection may be called to the following references :

Dr. W. Fischer, assistant to Professor Buschke, who writes for Buschke's Dermatological Clinic in the Rudolf Virchow Krankenhaus, Berlin-the very center of the clinical exploitation of "606:" Medizinische Klinik, Nov. 6, 1910, p. 1778.

Professor E. Lesser and Sanitätsrath Dr. O. Rosenthal, Berlin: Deutsche medizinische Wochenschrift, Nov. 17, 1910, pp. 2175 and 2176.

Professor Gaucher, successor to Fournier in St. Louis Hospital, Paris: Paris Letter, The Journal A. M. A., Dec. 10, 1910, p. 2074.

Professor E. Finger, Vienna: Wiener klinische Wochenschrift, Nov. 24, 1910, p. 1667.

72 Madison Street.

\section{New and Nonofficial Remedies}

The Following additional articles have Been accepted by the Councll on Pharmacy and Chemistry of the American medical Association. Their acceptance HAS BEEN BASED LARGELY ON EVIDENCE SUPPLIED BY THE MANUFACTURER OR HIS AGENT AND IN PART ON INVESTIGATION MADE BY OR UNDER THE DIRECTION OF THE COUNCIL. CRITICISMS AND CORRECTIONS ARE ASKED FOR TO AID IN-THE REVISION OF THE MATTER BEFORE PUBLICATION IN THE BOOK "New and Nonoffictal Remedies."

The CoUnCIL DESIRES PHYSICIANS TO UNDERSTAND THAT THE ACCEPTANCE OF AN ARTICle DOES NOT NECESSARILY MEAN A RECOMMENDATION, BUT THAT, SO FAR AS KNOWN, IT COMPLIES WITH THE RULES ADOP'TED BY THE COUNCIL.

\section{W. A. Puckner, Secretary.}

L-SUPRARENIN SYNTHETIC.-L-suprarenin synthetic is epinephrine produced synthetically according to the method of Stolz \& Flaecher (Ztschr. f. physiol. Chem., 58, p. 189).

$L$-suprarenin synthetic is a white odorless powder nearly insoluble in water, alcohol and ether. It melts at $211^{\circ}-212^{\circ}$. It has the power of rotating polarized light to the left:-

$$
[\mathrm{a}] \frac{19.6^{\circ}}{\mathrm{D}}=-\mathbf{5 1 . 4 ^ { \circ }}
$$

L-suprarenin synthetic has the chemical and physical properties and physiologic effect of natural epinephrine obtained from suprarenal glands.

Manufactured by Farbwerke vorm. Meister, Lucius \& Bruening, Hoechst a. M., Germany. (Victor Koechl \& Co., New York.) German patent No. 222,451.

L-SUPRARENIN SYNTHETIC BITARTRATE.-L-suprarenin synthetic bitartrate is the acid tartrate of 1-suprarenin synthetic.

It is a white odoriess powder readily soluble in water yielding an acid solution. It melts at $149^{\circ}$ and rotates polarized light to the left.

Manufactured by Farbwerke vorm. Meister, Lucius \& Bruening, Hoechst a. M., Germany. (Victor Koechl \& Co., New York). Ges'man patent No. 222,451 .

\section{PHARMACEUTICAL PREPARATIONS ACCEPTED FOR}

\section{N. N. $\mathbf{R}$.}

The following dosage forms of accepted proprietary articles have been accepted for N. N. R.:

Protan and Opium Tablets, No. 1.-Each tablet contains opium $0.005 \mathrm{Gm}$. (1/12 grain) and protan $0.162 \mathrm{Gm}$. (21/2 grains).

Protan and Opium Tablets, No. 2.-Each tablet contains opium $0.03 \mathrm{Gm}$. ( $1 / 2$ grain) and protan $0.5 \mathrm{Gm}$. ( $71 / 2$ grains).

Supracapsulin Inhalent.-A solution $1: 1,000$ of supracapsulin in an aromatized neutral oil, containing approximately 2.5 per cent of chloral and 10 per cent. of alcohol.

\section{Therapeutics}

\section{NOT TUBERCULOSIS}

History.-A young man, aged 28, came on Oct. 7, 1910, to ascertain how long he could live. He had been told that he had tuberculosis of the lungs, and had been treated three years for that disease. He had not worked for the last seventeen weeks, and was, in his opinion, rapidly growing worse. He had not a real cough, but he hawked and raised a great deal of yellowish sputum which was very frequently streaked with blood; in fact, hardly a day passed that he did not raise some blood. He had considerable gastric indigestion, some nausea, but no vomiting, quite a little gastric flatulence, but no pyrosis. A year and a half ago he had trouble with diarrhea, having movements of the bowels from ten to twelve times a day, but for the last six months the bowels had moved only two or three times a day, and not loose. The previous history shows that the patient had never really coughed, and that he had been subject to nose-bleed, sometimes as often as five or six times a week; it might not occur for several weeks, and then he would again have a period of it. He had no recognizable palpitation; no dyspnea on exertion; slept well; had had no swelling of the feet and legs. He had never had any urinary trouble, except a gonorrhea twelve years ago, and had no syph. ilitic history. He did smoke a great. deal, but not so much during the last year. He also chewed tobacco. He had never used much alcohol. The only severe illness he ever had was pneumonia when 12 years of age. The patient's father died of kidney trouble; his mother was alive and well. One sister died of tuberculosis three years ago. The patient did not live in the same house as this sister. Three sisters and two brothers were alive and well.

Examination.- Last year at this time the patient weighed 127 pounds; he now weighed 136 , and the weight had remained stationary for the last three months. His tongue was slightly coated and moist. The pulse was 78 , occasionally intermittent. 'Temperature was 98 at $2 \mathrm{p} . \mathrm{m}$. 'The heart was not enlarged, but showed an incomplete contraction of the ventricles every sixth to tenth beat, which accounted for the intermittency at the wrist. There were no murmurs. The lungs were absolutely normal. The abdomen was normal, with perhaps slight epigastric tenderness. There was an enlarged thyroid gland. The pharynx showed chronic catarrh, as did the naso-pharynx. The lingual tonsil was enlarged, coated, and showed a number of dilated blood-vessels. The nostril which bled the most frequently, the left, showed a swollen mucous membrane, and the mucous membrane of the septum was unusually red. The urine and the sputum were negative.

Diagnosis.-Disturbed thyroid secretion caused the diarrhea and the tendency to bleeding. The large amount of tobacco caused the throat catarrh, lingual tonsil irritation, and probably the irregular action of his heart. There was no tuberculous trouble in this patient.

Treatment.-The patient was told to go to work; to stop tobacco; and given Seiler's tablet gargle, and a small dose of sodium iodid to satisfy the probable need of the thyroid to improve its activity.

$\mathbf{R}$

Gm. or c.c

Sodii iodidi ................ 2

Aquæ menthæ piperitæ $\ldots \ldots \ldots \ldots \ldots 100 \mid$ or $\underset{\mathrm{H}\} \mathrm{jiv}}{\mathrm{gr}} \mathbf{x x x i}$

M. et Sig.: A teaspoonful, in water, three times a day, after meals. 